\title{
SCANNING TUNNELING MICROSCOPY AND ELECTROCHEMISTRY *
}

\author{
A.J. ARVÍA \\ Instituto de Investigaciones Fisicoquimicas Teóricas y Aplicadas (INIFTA), \\ Facultad de Ciencias Exactas, Universidad Nacional de La Plata, Sucursal 4, \\ Casilla de Correo 16, 1900 La Plata, Argentina
}

Received 1 August 1986; accepted for publication 15 September 1986

The experimental gap existing between surface science and heterogeneous chemical kinetics applies also to electrochemistry. Difficulties for modelling the electrode surface topography under equilibrium and non-equilibrium conditions, particularly for solid electrodes, are presented. Attention is focussed on structural problems of metal/solution interfaces encountered in corrosion and passivation of metals and in electrocatalysis, although the analysis also extends to metal electrodeposition and to semiconductor/solution interfaces. STM has already provided important imaging of preferred oriented platinum electrode surfaces as well as large surface area platinum electrodes exhibiting practically no diffusional and ohmic polarization effects. Possible applications of STM to underpotential deposition of metals and to chemically modified electrodes are envisaged among others. Some future perspectives of STM for electrochemistry are advanced.

\section{Introduction}

A few years ago the scanning tunneling microscope (STM) burst upon the scene [1]. The new instrument appears to offer the possibility of providing real space images of atomic structure, a fact of fundamental advantage for understanding the microscopic structure of matter. The rapid progress in STM design is accompanied by an explosive number of possible applications, particularly on general aspects of solid surfaces and species adsorbed on surfaces. The important feature of these research activities has been principally the involvement of physicists and chemical physicists, but also the interaction with electrochemists in the immediate future will result not only in information transfer but also will stimulate their interest in the electrochemical interface. The latter is more complex in general than the solid-vacuum interface and the atomically and molecularly sensitive techniques available for its characterization are considerably more limited.

\footnotetext{
* Invited lecture at STM'86, Santiago de Compostela, Spain, July 14-18, 1986.
} 
This lecture attempts to present an areal survey of some problems of electrochemistry where the future extended application of STM can be profitable. It is delivered firstly not for the electrochemists but for non-electrochemists in order to encourage them to improve STM principles and design for electrochemical experiments, although certainly it can be useful also to the electrochemical community for getting acquainted with this new kind of instrument which facinates scientists and non-scientists alike.

\section{Surface science, heterogeneous catalysis and electrochemistry}

Heterogeneous chemical reactions imply interactions between reactants and substrate which become of outmost importance in determining the yielding of the final product. A large number of heterogeneous reactions occur on solid substrates.

The rate of a heterogeneous reaction can be expressed as the product of two terms one depending on the concentration of reactant at the reaction interface, and another involving the energy assisting the chemical reaction, namely, thermal energy (thermal reactions), thermal and electric energy (electrochemical reactions), thermal and radiation energy (photochemical reactions, radiation chemistry), etc.

In electrochemistry the characteristics of the electrode surface plays a fundamental role in the kinetics of the reaction, particularly in those reactions involving the formation of adsorbed intermediates such as adatoms or adradi-

Table 1

Experimental gaps between surface science, heterogeneous catalysis and electrocatalysis

\begin{tabular}{|c|c|c|c|}
\hline Parameter & Surface science & Heterogeneous catalysis & Electrocatalysis \\
\hline Pressure & $10^{11}-10^{13}$. lorr $^{a)}$ & $10^{5}-10^{7} \mathrm{~Pa}$ & $10^{5}-10^{6} \mathrm{~Pa}$ \\
\hline Temperature & $\begin{array}{l}\text { About } 300 \mathrm{~K} \text { or } \\
\text { lower }\end{array}$ & $273-773 \mathrm{~K}$ & $250-1200 \mathrm{~K}$ \\
\hline Activation energy & Thermal & Thermal & $\begin{array}{l}\text { Thermal and } \\
\text { electrical }\end{array}$ \\
\hline $\begin{array}{l}\text { Degree } \\
\text { of dispersion }\end{array}$ & $\begin{array}{l}\text { Very low; films } \\
\text { and single } \\
\text { crystal planes }\end{array}$ & $\begin{array}{l}\text { Very high; sometimes } \\
\text { nearly atomic } \\
\text { dispersion }\end{array}$ & $\begin{array}{l}\text { Low, intermediate } \\
\text { and very high }\end{array}$ \\
\hline $\begin{array}{l}\text { Crystallographic } \\
\text { plane distribution }\end{array}$ & $\begin{array}{l}\text { Often single } \\
\text { crystal surfaces }\end{array}$ & polycrystalline surfaces & $\begin{array}{l}\text { None (liquid metals); } \\
\text { polycrystalline surfaces, } \\
\text { occasionally single } \\
\text { crystal surfaces }\end{array}$ \\
\hline Capacitance & Very small & Very small & Large \\
\hline
\end{tabular}

a) 1 Torr $=133.3 \mathrm{~Pa}$ 
Table 2

Some surface physical chemistry methods employed in electrochemistry

\begin{tabular}{ll}
\hline In-situ & Ex-situ \\
\hline Raman spectroscopy & Auger electron spectroscopy \\
Mössbauer spectroscopy & X-ray photoelectron spectroscopy \\
Reflection spectroscopies & UV photoelectron spectroscopy \\
Ellipsometry & Electron energy loss spectroscopy \\
Electron spin resonance & Low energy electron diffraction \\
X-ray diffraction & Work function measurements
\end{tabular}

cals. These electrochemical reactions can be considered as a particular type of heterogeneous chemical reactions. Therefore, in this case the term electrocatalysis $[2,3]$ gives a clear idea of the situation.

As one compares the experimental conditions usually employed in surface science, heterogeneous catalysis $[3,4]$ and electrochemistry [5] substantial differences can be noticed. This situation is clearly illustrated in table 1. Obviously, the experimental gap between surface science and catalysis (heterogeneous and electrocatalysis) turns difficult or even sometimes impossible to infer conclusions from surface science results applicable to more condensed systems are usually encountered in heterogeneous catalysis and electrocatalysis. Certainly, any attempt to shorten this experimental gap becomes extremely valuable. In this respect, for instance, recent research works on single crystal electrodes are quite relevant as for the first time experimental techniques initially developed for surface science studies were extended to electrochemistry for attempting a better understanding of the properties of the electrode/solution interface both under equilibrium and non-equilibrium conditions. Reviews on the matter are given in refs. [6-10]. For this purpose many surface physico-chemical methods either in-situ or ex-situ are being increasingly employed in electrochemistry (table 2).

\section{Some characteristics of electrochemical reactions}

The electrode/solution interface comprises a relatively extended region where the properties of the solution changes on moving along a direction perpendicular to the electrode surface outwards [5,11]. Changes in the electrode properties extend also from the electrode surface inwards for semiconductor electrodes [12]. The properties of the solution (composition, electroneutrality, chemical potential, density, etc.) in the neighbourhood of the electrode surface are changed as compared to the average bulk properties. For simple electrode geometries and defined hydrodynamic conditions [13] the greatest change of a particular property is usually confined up to a certain 
distance from the electrode surface, so that is becomes possible to associate the change of each property with a particular layer parallel to the electrode surface (electrical double layer, diffusional boundary layer, hydrodynamic boundary layer, etc.)

Electrochemical reactions imply two principal rate processes, namely, the transport of reactants from the bulk of the solution to the space region adjacent to the electrode surface through migration, diffusion and convection, and the proper electron transfer reaction occurring at the electrode. The rate of the electron transfer reaction is directly determined by the electric potential difference applied to the electrode, the characteristics of the electrode surface and the structure of the electrical double layer.

Electron transfer processes occur at the electrode/solution interface within a definite space region limited on one side by the electrode surface itself and on the other extending up to a thickness of the order of $1 \mathrm{~nm}$, that is within the inner part of the electrical double layer.

\subsection{The electrical double layer}

One of the properties which distinguishes the metal/vacuum and metal/gas interface from the metal/aqueous electrolyte interfaces is the electrical capacitance. Let us consider a $2 \mathrm{~mm}$ diameter gold sphere either in vacuum or in contact with 0.1 potassium chloride. For the former case the capacitance is $1.1 \times 10^{-13} \mathrm{~F}$, whereas for the latter $2.5 \times 10^{-6} \mathrm{~F}$. This large difference in the capacitance value is due to the space charge localized in the immediate vicinity of the metal in contact with the electrolyte solution. If $q^{\mathrm{me}}$ and $q^{\text {sol }}$ denote the electrical charge at the metal side and at the solution side, respectively, the electroneutrality condition for the entire system requires that:

$q^{\mathrm{me}}=-q^{\mathrm{sol}}$.

This means that the distribution of charges at the interface can be associated with a plane for one type of charge at the metal surface, and a different plane for the other type of charge placed at the solution side. Therefore, the electrical double layer at equilibrium on a smooth homogeneous surface can be represented as an electrical double layer.

\subsection{Simple structures for the electrical double layer}

The potential difference at the polarizable electrode/solution interface implies that the electroneutrality condition in the solution region immediately adjacent to the electrode surface, that is in the 0.1 to $10 \mathrm{~nm}$ range, is no longer fulfilled. The corresponding distribution of charges determines the structure of the electrical double layer region whose knowledge at the atomic level is of outmost importance in electrochemical kinetics. Most conclusions on the 
structure and properties of the electrical double layer were derived from studies made with the liquid mercury electrode under polarization equilibrium because it offers a clean smooth surface which behaves as an ideally polarizable electrode. The resulting data were treated following different approaches based either on classical thermodynamics [14], or structural models [15], or statistical thermodynamics and quantum mechanics [16].

The simplest structure of the electrical layer is expected in the absence of specific adsorption at the electrode surface. In this direction two models can be mentioned, namely the Helmholtz model [5] which corresponds to a parallel plate capacitor, and the Gouy-Chapman model [6] which takes into account the presence of ions in solution as point charges having a Boltzmann distribution and interacting with the electric field under elcctrostatic equilibrium. The potential energy which is exclusively electrostatic is only a function of the distance to the electrode.

\subsection{Adsorption at electrodes}

In contrast to the metal/gas interface the metal/solution interface offers, in principle, a larger number of specific interactions such as metal-neutral molecule, metal-ion, metal-solvent; ion-neutral molecule, ion-solvent, ionion; neutral molecule-neutral molecule, neutral molecule-solvent and solvent-solvent interactions. The balance of all these interactions should play a definite role in determining the type of complex competitive adsorption equilibria prevailing in each system. Nevertheless, for many electrochemical processes of technical interest one can distinguish the following two limiting cases: metal-ion adsorption and metal-molecule adsorption. In general, such adsorption processes can be desirable for some electrocatalytic reactions and corrosion inhibition or undesirable as they assist, for instance, the corrosion of metals.

The simplest structural model for the electrical double layer with the specific adsorption is that given by Stern [6] which admits that the charge at the solution side is made up of two contributions, one due to the proper solution constituents, $q^{\mathrm{d}}$, and another related to the adsorption charge, $q^{\text {ad }}$. Therefore, $q^{\text {sol }}$ is

$q^{\text {sol }}=q^{\mathrm{d}}+q^{\text {ad }}$.

The surface concentration of the adsorbed species, $i$, represented by $\Gamma_{i}$, is given by the following thermodynamic relationship for constant temperature $(T)$, pressure $(P)$ and chemical potential $(\mu)$ :

$\left(\frac{\delta \gamma}{\delta \mu_{i}}\right)_{T, P, \mu_{j(j \neq i)}}=\Gamma_{i}$

where $j$ denotes any solution component other than $i$. 


\subsection{The problem of water at the metal/solution interface}

Most structural electrical double layer models adopt the simple notion that each water molecule at the interface behaves as a single dipole. The interpreatation of the capacitance/potential relationship for a mercury electrode indicated the need of different models for the structure of water at the interface. In this direction a two-orientation model with up and down water molecules was proposed [17]. This model was later improved by including molecular aggregates either with a single or two different configurations involving either a fixed or an arbitrary number of water molecules per aggregate $[18,19]$. Finally, a complex water structure based upon that developed for different hydrates was also advanced [20]. However, despite the efforts already made the problem of the structure of water at the electrode/solution interface remains open [21] and further research is certainly required, especially through the application of in situ spectroscopic techniques.

\subsection{Orientation of adsorbed molecules at electrodes}

Another relevant problem related to the electrical double layer structure concerns the orientation of adsorbed molecules at electrodes. In this respect the situation can be summarized as follows [22]: Aromatic compounds adsorb, in principle, irreversibly. Some of them at low concentration adsorb in a planar form but at large concentrations a reorientation takes place which promotes the interaction between the borders of the molecules and the metal surface. In addition, the transition from planar to border adsorption is irreversible. Border structures occupying the minimum area are the most stable ones, particularly if other functions of the molecule are located far from the adsorbate-substrate interaction side. Other molecules such as antraquinones adsorb only in a planar form but bipheniles adsorb in a planar form at low concentration, a border form at intermediate concentration with the participation of the two rings, and at high concentrations as a flopping form involving a single ring. Compounds involving either heteroatoms or other functional active groups interact with the surface by orienting those atoms or groups towards the surface of the metal. An adsorption order for the different groups has been established [22]. In general, planar structures are favoured at low packing density but the adsorbate accumulation turns the planar structures into others occupying less area. The coadsorption or deposition of a foreign molecule when a planar structure exists on the surface turns the latter into a border structure.

Protein molecules are irreversibly adsorbed on metals [23]. This is accompanied by denaturalization and shape compression of the molecule to a thickness comprised between 0.8 and $1.0 \mathrm{~nm}$. 


\subsection{The real solid electrode surface under equilibrium conditions}

From the preceding sections one can conclude that even for smooth metal electrode surface/aqueous solution interfaces a completely satisfactory structural model for the electrical double layer is lacking. Most of the models available at present imply a continuous description of the metal surface and fairly drastic assumptions imposed to the structure of the solution side of the electrical double layer.

Even under equilibrium conditions most of solid electrode surfaces are actually non-homogeneous poorly defined polycrystalline materials [6,8]. Hence, there is a limitation for the application of simple structural models to real systems. Recent works on the electrical double layer at clean structurally well defined solid/liquid interfaces [7,24] have shown that each crystallographic surface involves a particular work function and correspondingly, a distinguishable potential of zero charge. For gold, silver and copper the potential of zero charge for the different low index crystallographic faces are assembled in table 3. A similar change is also reported for the work of extraction of electrons from copper single crystals as a function of orientation [25]. Therefore, one should expect that the metal-solvent and metal-ion interactions of each crystallographic face of the solid surface be different. In this case the polycrystalline solid surface can be imagined as a set of patches with different properties.

On the other hand, solid surfaces at room temperature even for single crystal structures include different types of defects which in turn represent distinguishable reacting sites in heterogeneous chemical reactions. Accordingly, the density of each type of defect constitutes a relevant parameter for a quantitative interpretation of kinetic data.

Finally, surface inclusions must also be considered. They can play an important role either in determining the density of surface defects or they become themselves new reacting sites at the solid surface. Various structural models and equivalent circuits which take into account the complexity of the real solid metal electrode surface have already be presented [26]. From the preceding consideration one concludes that STM appears as a promising technique for further understanding the electrical double layer structure by

Table 3

Potentials of zero charge (versus saturated calomel electrode) $\left(E_{z}\right)$ for silver, gold and copper single crystals in $0.01 \mathrm{M}$ sodium fluoride [10]

\begin{tabular}{llcl}
\hline Miller index & $E_{z}$ (silver) $(\mathrm{V})$ & $E_{z}$ (gold) $(\mathrm{V})$ & $E_{z}$ (copper) $(\mathrm{V})$ \\
\hline$(111)$ & -0.69 & 0.33 & -0.265 \\
$(100)$ & -0.91 & 0.14 & -0.29 \\
$(110)$ & -1.01 & -0.05 & $-0.315(-0.365)$ \\
\hline
\end{tabular}


taking into account that it can be routinely removed from its electrolyte essentially unchanged (emersed double layer) and studied in detail ex-situ [27].

\section{Non-equilibrium electrochemistry at solid metals}

The degree of advance of a chemical or electrochemical reaction is given through the corresponding rate equation. In principle, the operating conditions at the reacting system are shifted from those related to thermodynamic equilibrium. Hence, any electrical double layer structural data derived from the homogeneous, smooth, ideally polarizable metal/solution interface should be critically considered for their application to the reacting polycrystalline solid/solution interface.

To envisage the application of STM to electrochemical problems let us focus the attention on two processes which are representative for applied electrochemistry. One of them is related to the stability of materials (corrosion and passivation of metals) and another concerns electrochemical energy conversion and substance production (electrocatalysis).

\subsection{Problems arising from metal corrosion and passivation}

The comparison between the mercury $/ 1 \mathrm{M}$ sodium chloride interface and the corroding metal/aggressive solution interface allows one to determine common and differential aspects of these two electrochemical interfaces. Thus, among the common aspects one observes that both interfaces contain ions and molecules, and there is a potential difference across each interface, that is a certain electrochemical polarization exists, and under these circumstances different interactions become possible. Otherwise, outstanding differences between those two interfaces can be noticed. Thus, for the mercury/1M sodium chloride interface one deals with a double layer structure under equilibrium conditions. Conversely, the corroding metal/aggressive solution interface comprises usually a heterogeneous rough metal surface under nonequilibrium conditions where a reaction takes place in most cases in the presence of a continuous or discontinuous film.

Despite the large number of publications on this matter the present knowledge of the mechanism of corrosion of metals and their passivation, particularly at the atomic and molecular levels, is rather limited. As examples for iron see refs. [28]. However, the application of various surface physics techniques to the study of these processes in recent years has contributed to a real advance in understanding their kinetics [29].

Some of the problems arising from metal corrosion and passivation whose solution can be attempted through the application of STM can be summarized as follows:

(i) Structure of the electrical double layer under non-equilibrium. 
(ii) Determination of roughness characteristics.

(iii) Imaging the topography of the metal surface at the early stages of attack.

(iv) Mechanism of passive layer growth (2D and 3D nucleation and growth).

(v) Adsorption of aggressive anions and corrosion initiation.

(vi) Adsorption of inhibitors for corrosion.

(vii) Organic film formation and structure.

(viii) Structure and characteristics of passive layers as a function of the water content.

(ix) Ageing effects on passive layers.

It is clear that the development of non-destructive surfaces techniques sensitive to the atomic level and applicable in-situ are of the outmost importance for solving a great number of these intriguing problems. Certainly, the preceding analysis can be immediately extended to metal electrodeposition and electrocrystallization.

\subsection{Electrocatalysis}

Chemisorption at solid electrodes plays a very important role in electrocatalysis [2]. Let us pay attention to platinum electrodes in acid solutions as an example of electrodes which are being used in electrochemical energy conversion devices [30].

Studies carried out on both single crystal and polycrystalline platinum in different aqueous electrolytes indicate the existence of large adsorption energy regions (potential windows) for hydrogen and oxygen adatoms resulting from the underpotential decomposition of water molecules [31,32]. There is little doubt that the corresponding reactions imply a complete electron transfer with the formation of a chemical bond. Each pair of voltammetric peaks for hydrogen adatom electroadsorption/electrodesorption has been attributed to a particular crystallographic face of platinum. The structure of those peaks depends substantially on the nature and concentration of electrolyte, a fact which suggests the overlapping of the potential ranges related to anion adsorption and hydrogen adatom electroadsorption [32]. Furthermore, the different electroadsorption/electrodesorption processes also promote the metal surface restructuring [33]. These characteristics of the hydrogen adatomplatinum interactions extend, in principle, to any electrocatalyst-adsorbate system. Therefore, the selection of an electrocatalyst for a particular reaction results, among others [3], from three fundamental features, namely, the degree of specificity of the material for the reaction; the yielding of product; and the possibility of designing electrodes comprising a large active surface area. At present these three requirements are met through empirical or semi-empirical procedures, although the trend is to work out conclusions from basic principles of solid state physics and chemical kinetics. Certainly, this purpose can be 
achieved through a clear understanding and handling of the electrocatalyst/solution interface at the atomic level.

Let us consider the contribution of STM to understand two main problems commonly found in heterogeneous catalysis and electrocatalysis which refer to the identification and distribution of reacting sites, namely, those properties of the surface directly related to the specificity of the reaction, and the evaluation and modelling for the increase in active surface area as the rate of the reaction is directly proportional to the accessible active surface area. Obviously, the specificity of the electrocatalyst should depend on whether one deals with polycrystalline, single crystal or faceted surfaces. Most reliable data on the matter were recently obtained through the preparation of well defined surfaces, and surface structure characterization by applying reflection electron diffraction and electron spectroscopies combined with efficient commercial ultrahigh vacuum equipment. A generous number of articles dealing with this subject will be found in ref. [6]. As far as the topography of platinum is concerned the advances very recently made through the application of STM are important $[34,35]$.

\subsubsection{STM and the topography of preferred monoriented platinum}

Preferred monoriented platinum surfaces of either (110), (100) or (111) type crystallographic orientation can be developed either from single crystal or polycrystalline structures by subjecting the starting material in acid electrolyte to a fast periodic potential of the order of a few $\mathrm{kHz}$, covering the potential range where the hydrogen and oxygen adatom electroadsorption/electrodesorption takes place [36]. The characteristics of the resulting surface depends on the upper and lower potential limits and frequency of the fast periodic potential signal, and they can be followed through the electrochemical adsorption/desorption spectrum for hydrogen adatoms [10,36]. A full account of these experiments is given in ref. [35] *. These STM results offer for the first time the topography of platinum electrode surfaces on the nanometer scale, which shows the growth of oriented corrugations and the development of steps and terraces in different domains of the surface.

\subsubsection{Modelling for the increase in active surface area through STM imaging}

Usually in dealing with the electrocatalyst active surface area several concepts such as macro- and micro-roughness, and particle size distribution in dispersed metals are referred to. As fas as active surface area and reactivity is concerned the macro- and micro-pore geometry and distribution are important characteristics [37]. An electrocatalyst such as platinum can be used either as

* This work was developed at the Laboratorio de Fisica Fundamental, Universidad Autónoma de Madrid, with samples provided by División Electroquímica del INIFTA, Universidad Nacional de La Plata. 
polished or large active surface area electrode. The latter can be employed as a dispersed metallic electrode involving negligible diffusional and ohmic resistances [38].

STM has already been successfully employed to study the structure of porous platinum electrodes which are produced by firstly growing a relatively thick hydrous platinum oxide layer in acid solution under a fast periodic potential signal ranging from a low potential limit of about $0 \mathrm{~V}$ (versus normal hydrogen electrode) up to about $2 \mathrm{~V}$, and secondly, by electroreducing the thick hydrous oxide layer by means of a slow potential scan [38].

The model for describing the behaviour of these electrodes, as derived from STM data, consists of a pile of pebble-like platinum clusters of about $10 \mathrm{~nm}$ diameter which originates an inner channel-likc structure permitting that practically the entire surface of each pebble becomes accessible for reactants * A full account of these results is given elsewhere [35].

\subsubsection{Present status of STM for electrocatalysis}

At present the use of STM for determining the characteristics of electrocatalyst surfaces offers pros and cons which can be summarized is the following way. The technique results of extremely high resolution reaching the atomic level, it introduces a negligible potential perturbation so that no harmful action on the specimen is practically made. It allows to work under vacuum or controlled atmosphere; it offers the possibility to follow changes produced through temperature variation, and it is capable of supplying topographical and spectroscopic information of a new order for understanding chemical interactions. Conversely, it presents difficulties which are proper to an ex-situ technique, and for increasing the reliability of data further theoretical and experimental work on the tip design is desirable. Continuing efforts for direct tip operation in solid/liquid and solid/solution interfaces are encouraged.

\section{Modification of surface properties of metals through underpotential deposi- tion}

It is well established that a great number of metals can be electrodeposited on a conducting substrate either at the monolayer or submonolayer level through underpotential deposition (upd). Reviews on the subject are given in refs. [39]. These processes have been extensively studied for both polycrystalline and single crystal metallic surfaces. Examples are the upd of $\mathrm{Cu}, \mathrm{Sn}, \mathrm{Tl}$, $\mathrm{Pb}, \mathrm{Bi}$ and $\mathrm{Hg}$ on platinum and gold. Under these conditions the properties of

* This work was made at the Universidad Autónoma de Madrid with samples prepared in part at the same University and in part at the University of La Plata. 
the surface for different reactions are substantially changed, a fact which turns these modified electrodes very promising.

In principle, one should expect that a technique such as STM presents an important tool for providing data on the structure of upd layers as a function of coverage; the modification and restructuring of the substrate caused by the electrodeposited species; the formation of alloys at the surface level; the possible change from $2 \mathrm{D}$ to $3 \mathrm{D}$ configuration; and the mechanisms involved in all those processes. Up till the present indirect information of these aspects of upd of metals was obtained through electrochemical measurements [40].

\section{Chemically modified electrodes}

In recent years it was found that the catalytic activity of electrodes can be modified by anchoring different atoms or molecules at the surface. Extensive revisions on the subject are given elsewhere [41]. This new kind of electrodes denoted as chemically modified electrodes, involve the attachment of molecules to the surface probably through either a direct contact or through water molecules. The structure of these electrodes covered with organic molecules can likely be assimilated to that of a surface covered by an immobilized membrane which can be loaded with different redox systems for assisting or hindering certain electron and proton transfer processes.

Chemically modified electrodes present a large number of questions which have not yet been satisfactorily answered and the application of surface imaging techniques at the atomic level appears very promising for providing additional basic information. As examples of some of those questions it can be noticed that no reasonable explanation for the number of chemical bonds and reacting sites at the surface as well as their distribution can be advanced. Likewise, the average configuration and stability of the entire structure is rather poorly known. The same applies to homogeneity, permeability, island and crosslinked structures and their stability throughout time.

\section{Outlook on possible applications of STM in heterogeneous catalysis and electrochemistry}

Possible applications of STM in heterogeneous catalysis and electrochemistry can be briefly described as follows:

(i) Determination of local surface topography at the atomic level and surface spectroscopy of a new order.

(ii) Kinetics of restructuring and ageing processes at surfaces.

(iii) Study of adsorbate-substrate interactions.

(iv) Imaging data which can be related to the double layer structure at solid electrodes under equilibrium and non-equilibrium conditions. 
(v) Extension to surface spectroscopy measurements. Correlation between STM and SERS data.

(vi) Possible coupling between STM and SEM techniques.

In conclusion, there is a promising future for the applications of STM in all fields of heterogeneous catalysis and electrochemistry through the new look it offers for the surface of condensed matter. Future developments, particularly those involving possible in-situ measurements, and coupling with other surface techniques will extend the capability of STM.

\section{Acknowledgement}

The author thanks Professor N. García and the Organizing Committee for the invitation to participate in STM'86.

\section{References}

[1] G. Binnig, H. Rohrer, G. Gerber and E. Weibel, Phys. Rev. Letters 50 (1983) 120;

G. Binnig and H. Rohrer, Sci. Am. 253 (1985) 50.

[2] W.T. Grubb, Nature (London) 198 (1963) 883.

[3] G.P. Sakellaropoulos, Advan. Catalysis 30 (1981) 217;

A.J. Arvia and M.C. Giordano, Electrocatálisis, Aspectos Fundamentales (FECYC, Buenos Aires, 1983).

[4] T.N. Rhodin and G. Ertl, Eds., The Nature of the Surface Chemical Bond (North-Holland, Amsterdam, 1979).

[5] J.O'M. Bockris and A.K.N. Reddy, Eds., Modern Electrochemistry, Vol. 2 (Plenum, New York, 1970).

[6] A.T. Hubbard, Accounts Chem. Res. 13 (1980) 177.

[7] R. Parsons, J. Electroanal. Chem. 118 (1981) 1.

[8] A. Hamelin, Elektrokhimiya 18 (1982) 1413.

[9] A. Hamelin, J. Electroanal. Chem. 142 (1982) 299;

A. Hamelin, T. Vitanov, E. Sevastyanov and T. Popov, J. Electroanal. Chem. 145 (1983) 285

[10] R.M. Cerviño, W.E. Triaca and A.J. Arvia. Electrochim. Acta 30 (1985) 1323.

[11] J. Albery, Electrode Kinetics, Oxford Chemistry Series (Clarendon, Oxford, 1975) p. 55.

[12] H. Gerischer, in: Advances in Electrochemistry and Electrochemical Engineering, Vol. 1, Eds. P. Delahay and C. Tobias (Interscience, New York, 1961) ch. 4.

[13] V.G. Levich, Physicochemical Hydrodynamics (Prentice-Hall, Englewood Cliffs, NJ, 1962).

[14] P. Delahay, Double Layer and Electrode Kinetics (Interscience, New York, 1965).

[15] J.O'M. Bockris, B.E. Conway and E. Yeager, Eds., Comprehensive Treatise of Electrochemistry, Vol. 1, The Double Layer (Plenum, New York, 1980).

[16] S. Romanowski, W. Stasiak and L. Vojtczak, Electrochim. Acta 27 (1982) 511;

J.P. Badiali, M.L. Rosimberg, F. Vericat and L. Blum, J. Electroanal. Chem. 158 (1983) 253; A.L.G. van den Eeden, J.H. Sluyters and J.H. van Lenthe, J. Electroanal. Chem. 171 (1984) 195.

[17] R.J. Watts-Tobin, Phil. Mag. 6 (1961) 133:

N.F. Mott and R.J. Watts-Tobin, Electrochim. Acta 4 (1961) 79.

[18] B.B. Damaskin and A.N. Frumkin, Electrochim. Acta 19 (1974) 173;

R. Parsons, J. Electroanal. Chem. 59 (1975) 229;

S.Kh. Ait'yan, V.A. Benderskii, G.I. Volichko, V.S. Markin and Yu.A. Chizmadzhev, Soviet Electrochem. 19 (1983) 412. 
[19] S.L. Carnie and D.Y.C. Chan, Advan. Colloid Interface Sci. 16 (1982) 81.

[20] M.I. Florit, M.E. Martins and A.J. Arvia, J. Electroanal. Chem. 151 (1983) 209;

M.I. Sosa and A.J. Arvia, Anales Acad. Nac. Cienc. Exact., Fis. Nat. Buenos Aires 34 (1982) 153 .

[21] S. Trasatti, Electrochim. Acta 29 (1984) 1503.

[22] A.T. Hubbard, J.L. Stickney, P.P. Soriaga, V.K.F. Chia, S.D. Rosasco, B.C. Schardt, T. Solomun, D. Song, J.H. White and A. Wieckowski, J. Electroanal. Chem. 168 (1984) 43.

[23] B.A. Kuznetsov and G.P. Shumakovich, Soviet Electrochem. 20 (1984) 137.

[24] G. Valette and A. Hamelin, J. Electroanal. Chem. 45 (1973) 301.

[25] L. Peralta, Y. Betthier and J. Oudar, in: 4th Colloq. Phys. Chim. Surfaces Solides, Le Vide (1978) p. 83.

[26] I.A. Bagotskaya, B.B. Damaskin and M.D. Levi, J. Electroanal. Chem. 115 (1980) 189; M.A. Vorotyntsev, J. Electroanal. Chem. 123 (1981) 379.

[27] W.N. Hansen, C.I. Wang and T.W. Humpherys, J. Flectroanal. Chem. 90 (1978) 137;

D.M. Kolb and W.N. Hansen, Surface Sci. (1979) 205; J. Electroanal. Chem. 100 (1979) 493;

W.N. Hansen, Surface Sci. 101 (1980) 109;

W.N. Hansen, D.M. Kolb, D.L. Rath and R. Wille, J. Electroanal. Chem. 110 (1980) 369;

D.L. Rath and D.M. Kolb, Surface Sci. 109 (1981) 641;

W.N. Hansen, J. Electroanal. Chem. 150 (1983) 133.

[28] K.E. Heusler, in: Encyclopedia of the Electrochemistry of the Elements, Vol. 9A, Ed. A.J. Bard (Dekker, New York, 1982) p. 229;

B. Folleher and K.E. Heusler, J. Electroanal. Chem. 180 (1984) 77;

A.J. Arvia, in: Proc. 8th Intern. Congr. on Metallic Corrosion, Vol. III (1981) p. 2065.

[29] D.T. Larson, Corrosion Sci. 19 (1979) 657;

D.B. Williams, J. Metals (1980) 16.

[30] J.O'M. Bockris and S. Srinivasan, Fuel Cells: Their Electrochemistry (McGraw-Hill, New York, 1969).

[31] R. Faure, G. Guinet and R. Durand, J. Electroanal. Chem. 107 (1980) 205.

[32] J. Clavilier, J. Electroanal. Chem. 107 (1980) 211.

[33] R. Parsons, J. Electroanal. Chem. 150 (1983) 51;

F.T. Wagner and P.N. Ross, Jr., J. Electroanal. Chem. 150 (1983) 141.

[34] J. Gómez, L. Vázquez, A.M. Baró, N. Garcia, C.L. Perdriel, W.E. Triaca and A.J. Arvia, Nature, in press.

[35] L. Vásquez, J. Gómez, A.M. Baró, N. García, M.L. Marcos, J. González Velasco, J.M. Vara Cuadrado, A.J. Arvia, J. Presa, A. Garcia y M. Aguilar, J. Am. Chem. Soc,, submitted.

[36] R.M. Cerviño, W.E. Triaca and A.J. Arvia, J. Electrochem. Soc. 132 (1985) 266;

J.C. Canullo, W.E. Triaca and A.J. Arvia, J. Electroanal. Chem. 175 (1984) 337;

A.J. Arvia, J.C. Canullo, E. Custidiano, C.L. Perdriel and W.E. Triaca, Electrochim. Acta, in press.

[37] K. Scott, J. Appl. Electrochem. 13 (1983) 709;

J. Lesinski, G. Szymanski and L. Werblan, J. Electroanal. Chem. 205 (1986) 91;

T. Kessler, A.M. Castro Luna, W.E. Triaca and A.J. Arvia, J. Appl. Electrochem., in press.

[38] A.C. Chialvo, W.E. Triaca and A.J. Arvia, J. Electroanal. Chem. 146 (1983) 93; Anales Asoc. Quim. Arg. 73 (1985) 23.

[39] W.J. Lorenz, H.D. Herrmann, N. Wüthrich and F. Hilbert, J. Flectrochem. Soc. 121 (1974) 1167;

D.M. Kolb, in: Advances in Electrochemistry and Electrochemical Engineering, Vol. 11, Eds.

H. Gerischer and C.W. Tobias (Wiley, New York, 1978) p. 125;

G. Kokkinidis, J. Electroanal. Chem. 201 (1986) 217.

[40] E. Schmidt and H. Siegenthaler, J. Electroanal. Chem. 150 (1983) 59.

[41] A.J. Nozik, Ann. Rev. Phys. Chem. 29 (1978) 189;

R.W. Murray, Advan. Electroanal. Chem. 13 (1984) 191 